\author{
DEPARTAMENTO DE PARASTTOLOGIA DA FACULDADE DE MEDICINA DA \\ UNIVERSIDADE DE SÃO PAULO \\ (Diretor: Prof. Dr. SAMUel B. Pessôa)
}

\title{
NOTA SÔBRE A INCIDÊNCIA DE PORTADORES DE CISTOS DE E. HISTOLYTICA, NUMA COMUNIDADE RURAL (1) (2)
}

\author{
A. D. FRANCO DO AMARAL
}

Assistente

\section{INTRODUÇÃO}

O problema da amebiase, em nosso País, a-pesar-de contribuições valiosas a seu respéito, não está completamente elucidado, pois ainda não dispomos de dados suficientes para aquilatar da importância que essa protozoose deve assumir entre nós. Os estudos até agora levados a cabo em relação ao assunto, ainda que bem conduzidos, são relativamente escassos para a nossa vastidão territorial. Além disso, há discrepâncias nos resultados a que têm chegado vários pesquisadores.

Ao lado de investigações demonstrando que o parasitismo pela $E$. histolytica deve contribuir com um coeficiente elevado para os nossos quadros nosológicos, outras existem que, antes, levariam à crença de não ter esta parasitose tanta importânciạ, entre nós.

Passemos assim em revista algumas pesquisas que comprovam o que acabamos de afirmar.

Young (1), trabalhando na Amazonia, em 1922, fez um estudo sôbre os protozoários intestinais do homem, em Manaus, e levantou a percentagem de incidência de portadores de cistos' de E. histolytica em soldados das tropas federais aí acantonadas e em crianças de idade escolar; da mesma localidade. Nas tropas federais, o exame de 251 indivíduos revelou a incidência de $27,5 \%$ de portadores de cistos de $E$. histolytica, sendo $17,9 \%$ de cistos de mais de 10 microns e $11,5 \%$ de cistos de menos de 10 microns (havendo 5 casos infectados ao mesmo tempo por cistos grandes e cistos pequenos) ; em crianças de idade escolar, examinadas em número de 249 , foi obtida a percentagem de $22,5 \%$, com 14,0\% apresentando cistos maiores de 10 microns e 9,1\%

(1) Lido na reunião de 4-5-1942, da Sec. de Hig., Mol. Trop. e Inf., da Ass. Paul. de Medicina.

(2) Este trabalho foi feito sob os auspícios da Comissãa de Estudos da Leishmaniose do Estado de $S$ Pasilo. 
apresentando-os abaixo dessa dimensão, havendo 3 casos com infecção dupla. E, se bem que a incidência obtida pelo autor tenha sido elevada, não representaria ela a realidade, pois, conforme êle mesmo afirma, "One stool only was examined in each case so that only a fraction of the total infections are represented in the results".

Assinalemos, de passagem, que, tendo nós iniciado um inquérito entre soldados de tropas federais acantonadas em São Paulo, para estudar a incidência de helmintos e protozoários intestinais, deparamos também um coeficiente elevado de portadores de cistos de E. histolytica. Esse inquérito, por motivos alheios à nossa vontade, não pode ser prosseguido. Fornecemos, porém, os dados obtidos com os poucos casos que pudemos examinar, para cotejo com os obtidos por Young em indivíduos da mesma categoria dos nossos, isto é, elementos pertencentes às nossas tropas federais. Assim, nos 52 soldados que foram submetidos a um só exame de fézes pelo processo da centrifugação e flutuação no sulfato de zinco, de Faust, et al. ( $\mathcal{L}$ e 3 ), foram encontrados 15 com cistos de $E$. histolytica, o que equivale a 28,8\%, percentagem, como vemos, próxima da obtida por Young, em Manaus. Destes 15 indivíduos, 11 apresentavam cistos de mais de 10 microns, entre 10 e 15,6 microns; 1 apenas apresentava-os abaixo de $10 \mathrm{mi}$ crons, entre 7,8 e 9 microns; em 3 casos não foram os cistos medidos.

Em trabalho ainda en publicação, lido perante a Sociedade de Gastro-Enterologia e Nutrição de São Paulo, na reunião de março do corrente ano, apresentámos, em colaboração com C. D. de Avila Pires (4), um estudo feito para determinar a incidência de portadores de cistos de E. histolytica no total de 300 defentos da Penitenciaria de São Paulo. O processo de exame foi ainda o da centrifugação e flutuação no sulfato de zinco. $O$ método, porém, não foi aplicado uma só vez, mas repetido várias vezes entre os negativos, de acôrdo com o seguinte plano: os negativos ao primeiro exame foram submetidos a um segundo; os ainda negativos ao segundo foram submetidos a um terceiro; e assim por diante, até não mais revelar o exame coprológico portadores de cistos da endameba patogenica. Foram recessários cinco exames para descobrir todos os casos positivos para os cistos em questão; o sexto exame não mais os revelou. Assim, o estudo em aprêço, entre os 300 presidiários, nos requereu 1310 exames de fezes, fornecendo-nos a percentagem de $39,3 \%$ de portadores de cistos de E. histolytica. O primeiro exame, pelo citado processo, nos forneceu apenas $21,3 \%$ positivos. Esta incidência foi crescendo com os demais exames, até atingir a percentagem mencionada, - 39,3. O método de Faust, et al., pois, aplicado uma só vez, nos forneceu um pouco mais de $50 \%$ do total dos positivos. Esta investigação, realizada entre os presidiarios, aproveitou apenas os individuos aparentemente normais, na ocasião do exame, com fézes formadas, não catarrais. A incidência de portadores de cistos de E. histolytica obtida foi elevada, - 39,3\%, - como vemos. Um pouco mais da metade desses nossos examinados (157) era constituida de homens que, quando em liberdade, eram lavradores, provenientes, pois, do nosso meio ru- 
ral, e que nos forneceram um coeficiente de positividade para $E$. histolytica de 45,23\%; os restantes examinados (143), exercendo, quando libertos, profissões mais peculiares aos meios urbanos (operários, artifices, comerciários, funcionários públicos, musico, manipuladores de alimentos, etc.), nos deram a incidência de 32,87\%. Releva assinalar que essa elevada percentagem de portadores de cistos de E. histolytica, entre os nossos examinandos da Peninteciaria de São Paulo, não foi devida a uma contaminação intra presidio, segundo pudemos, com segurança, averiguar.

Ha ainda, na literatura medica nacional, uma série de outras pesquisas, levadas a cabo com o fito de determinar a etiologia das disenterias, sindromes disenteriformes ou pertubações intestinais, entre nós, das quais se evidencia a percentagem apreciavel com que a $E$. histolytica entra como agente causal de tais estados mórbidos. Em relação a São Paulo, Ficker (5) encontrou a E. histolytica em $35 \%$ dos casos de disenteria que examinou, o que o levou a afirmar ser principalmente de origem amebiana a disenteria observada em São Paulo. Pessôa e Corrêa (6) encontraram a endameba patogenica em 36 de 216 indivíduos $(16,6 \%)$ afetados de perturbações intestinais ou apresentando mau estado geral (sendo o exame coprológico feito para completar ou facilitar um dignóstico). Borges Vieira e Fleury da Silveirá (7), num total de 373 casos com perturbações intestinais, encontraram 137 parasitados pela E. histolytica ( $34 \%)$.

Em relação a outros pontos do Territorio Nacional, ha, por exemplo, um trabalho de Moniz Aragão (8), que, em João Pessôa, em 710 casos de sindromes disenteriformes, relacionou-se 168 vezes à presença de $E$. histolytica, o que dá a percentagem de 23,6 \%.

Ary Bento da Costa, citado por Cesar Pinto (9), em Santa Maria (Estado do Rio Grande do Sul), em 1370 exames de fézes, realizadios de 1935 até março de 1941, encontrou cistos e formas vegetativas de E. histolytica em $47,5 \%$ dos casos suspeitos.

Temos aí, pois, uma série de trabalhos pelos quais se pode avaliar, não só a grande dissemịnação da $E$. histolytica, em nosso País, como ainda a sua importância na etiologia das sindromes disenteriformes e perturbações intestinais.

Em contraposição, porém, como, aliás, já frisamos, outras pesquisas existem, das quais se inferiria não ser a incidência desse prôtozoário muito elevada entre nós.

Assim Genesio Pacheco (10), em 1928, contrariando a opinião de Brumpt, que estimou em 100000 o número de portadores de cistos tetra-nucleados, na cidade do. Rio de Janeiro, poz em prática o método preconizado pelo professor francês, para pesquisa de cistos de protozoários, com intuito de averiguar se a percentagem dos portadores de cistos tetra-nucleados aumentava. 'Tal fato, porém, não se verificou, pois, em 220 amostras de fézes, não catarrais, de indivíduos habitantes de zonas suburbanas e rurais do Rio de Janeiro, os cistos em questão compareceram apenas 4 vezes ou en 1,8\%. Esta incidência é bastante baixa, sobretudo se a compararmos com os nossos resultados, 
entre os presidiários da nossa Penitenciaria. Nesse inquérito, como vimos, examinamos, entre outros, 157 indivíduos procedentes de zonas rurais do Estado de São Paulo, em parte, pois, um grupo de inclivíduos em condições semelhantes às dos exaninados por Pacheco, cujo inquérito se realizou entre 220 individuos de zonas suburbanas e rurais do Rio de Janeiro. A nossa percentagem, entre esses 157 lavradores, sé elevou a 45,23 .

Ponto que também tem merecido a atenção dos nossos pesquisadores é o da incidência da $E$. histolytica, entre crianças. Aqui ainda, porém, não ha concordância nos coeficientes obtidos.

Marques da Cunha (11), em 1928; fez um estudo entre crianças no Rio de Janeiro. Diz o autor citado: "podemos desde logo assinalar que pelo menos grande parte das fezes examinadas eram de individuos que não apresentavam disturbios intestinais". Acrescenta: "A presença de quistos de Entamoeba com 4 nucleos, foi pesquizada com especial cuidado. tra-los".

Nos 805 casos examinados, somente 5 vezes podemos encon-

"A porcentagem de individuos, portadores de quistos de Entamoeba é pois, de acordo com a nossa estatistica de 0,62\%. Ainda que se admita que os resultados obtidos com un exame unico representem a terça ou quarta parte da percentagem real, teriamos $1,86 \%$ a $2,48 \%$ o que fica muito aquem dos $10 \%$ admitidos por Brumpt".

Lara e Carvalho (12), trabalhando em São Paulo, em 1936, dizem que, não obstante tivessem já a impressão clínica de ser a $E$. histolytica rara entre nós, nunca julgaram que a sua incidência fosse tão baixa, como a obtida na investigação que empreenderam. "Encontramos entre as crianças examinadas, unicamente, um pre-escolar que era portador de amebas $(0,62 \%$ dos casos positivos e $0,33 \%$ do total de parasitos".

Estes dados de Marques da Cunha e de Lara e Carvalho se afastam muito dos obtidos por Young, em 1922, em Manaus, entre 249 crianças de idade escolar, que forneceram a incidência de $22,5 \%$ de portadores de cistos de E. histolytica. Mais recentemente, Gomes de Morais (13), em 1939, fez um inquérito coprológico entre escolares, de 7 a 14 anos, em Florianopolis, examinando 1000 amostras de fezês. Segundo se depreende do seu trabalho, o autor não considera ainda resolvida a questão da unidade, dualidade ou pluralidade das Endamoeba produtoras de cistos de 4 nucleos. Todavia, julga acertado considerar, no trabalho citado, "todos os quistos tetranucleados como responsaveis potenciais pela disseminação da amebiose e encara-los como de importancia sob o ponto de vista sanitario". Considerando, assim, os cistos tetra-nucleados que encontrou como sendo de E. histolytica, estes, entre os escolares de Florianopolis, incidiram em $7 \%$ dos casos, pois 70 das amostras fecais examinadas os apresentaram. Diz mais Gomes de Morais que, admitindo que "esta taxa não corresponde á realidade, pois um unico exame condiciona sempre um erro sensivel, sendo aconselhado por alguns autores (Brumpt, Dobell, 
Carter, etc.) multiplicar a taxa encontrada por 3", os seus resultados dariam a percentagem de $21 \%$, no material examinado, resultados muito aproximados dos obtidos por Young, entre as crianças de Manaus. Mesmo que se considere que as pesquisas tenham sido levadas a cabo em pontos diversos do Territorio Nacional, fere sobremaneira a disparidade entre os resultados de Young e Morais, de $\mathrm{s}$ m lado, e Cunha e Lara e Carvalho, do outro.

Ressalta do exposto que ha muita discordância entre os autores que se têm ocupado da incidência da $E$. histolytica, em nossa população. Não ha negar que ainda são muito escassos os estudos epidemiológicos sôbre a amebiase em todo o País. E cremos, pois, que asṡiste razão a Faust (14), quando, em trabalho recente, diz: "Such scant information provides practically no basis for estimating the extent or of evaluating the significance of amebiasis in Brasil". Assim, só a multiplicação de inquéritos epidemiológicos, em várias regiões, em diversas categorias sociais, é que poderá trazer a verdade sôbre esse problema, entre nós.

Em nossa visinha Republica Argentina, os trabalhos magistrais de Greenway e Castex (15), cobrindo cerca de duas decadas de estudos continuados, vieram demonstrar a larga disseminação da $E$. histolytica em sua população. Na America do Norte, inquéritos bem conduzidos puzeram, por sua vez, em evidência a importância da protozoose para o País, a ponto de ser considerada "um perigo nacional" (16) e um "problema nacional" (17). Cremos que aqui, como alhures, a amebiase deve assumir uma significação que algumas investigações parecem querer negar. Ass nossas condições sanitárias, particularmente as dos nossos meios rurais, são bastante precarias para nos porem a coberto de tão serio problema.

Para o fim de contribuir com uma parcela para o estudo da amebiase, entre nós, vimos ha já algum tempo encarando o problema sob alguns aspectos.

\section{MATERIAL DE ESTUDO}

Quando de sua chefia na "Comissão de Estudos da Leishmaniose", sugeriu-nos o Prof. Samuel B. Pessôa que, em vista das facilidades, então existentes, de transporte para o interior, fizessemos um estudo minucioso sôbre o problema da amebiase numa comunidade rural. Extinta a referida Comissão, esse estudo não pode ser levado a cabo na integra. Não nos sendo possivel fazer um estudo completo a respeito da amebiase em nosso meio rurál, limitamo-nos a relatar nesta nota uma parte do trabalho que então idealizámos, isto é, o levantamento do índice, de portadores de cistos đe E. histolytica, no refericlo meio.

A localidade escolhida para esse inquérito foi o Núcleo Colonial "Barão de Antonina", situado no Municipio de Itaporanga.

O material a examinar procedia da localidade em questão conservado em soluto de formol a $10 \%$. 
A conservação dos cistos de protozoários na referida solução não conta com a simpatia de alguns pesquisadores, que alegam que tal conservador não deixaria os cistos em bôas condições para diagnóstico. Faust (op. cit.) afirma, a tal respeito: "It is of course essential that the stool specimen be fresh, if accurate diagnosis is to be made. It may be kept on ice to prevent putrefaction, but can not be satisfactorily preserved in formaldehyde, alcohol or phenol". A nossa experiência em tal sentido, sôbre a qual pretendemos ainda fazer uma comunicação especial, é que se, na verdade, o material fresco oferece maiores vantagens para o exame, o conservado em formol, entretanto, dá resultados satisfatorios. Marques da Cunha (11) julga que a conservação de cisto em formol dá resultados satisfatorios: "Os quistos assim conservados ha perto de $\mathbf{u m}$ ano, apresentam estrutura nitida embora se note em muitos deles un aspecto granuloso do plasma que não existia anteriormente". Rosenfeld (18) declara: "Podemios acrescentar ainda, que em algumas ocasiões, quando o exame não podia ser feito a tempo, mandamos fixar o material logo depois de emitido em 2 a 3 volumes de solução fisiologica com $10 \%$ de formol comercial neutralisado e o exame, praticado dias depois, permitia reconhecer as formas vegetativas com certa dificuldade, mas quanto aos quistos, a pesquiza é tão facil quanto nas fezes recentes. O material assim fixado, permanece em boas condiçôes durante anos, si tivermos a precaução de decantar o liquido fixador depois de deixar o material em repouso por alguns dias, e substituirmos por igual volume de uma solução nova que não precisará ser renovada".

O material conservado em formol, chegado ao laboratório, era submetido ao processo de concentraçãe de Faust et al. ( 2 e 3 ) e, em seguida, examinado mediante a coloração pela iodina, preparada de acôrdo com a técnica de D’Antoni (19).

\section{RESULTADOS OBTIDOS}

Em nosso inquérito foram examinadas as fézes de 400 indivíduos, entre os quais foram encontrados 64 portadores de cistos de $E$. histolytica, o que dá a incidência de $16 \%$. Considerando que, segundo nossas verificações com Avila Pires (op. cit.), o método de Faust et al., aplicado una só vez, num determinado grupo de indivíduos, revela apenas um pouco mais de $50 \%$ dos portadores de cistos de E." histolytica, é de crer que, no grupo em questão, os $16 \%$ se elevariam, no mínimo, a $32 \%$, caso fossem os exames repetidos, entre os negativos, várias vezes de acôrdo com o esquema que seguimos no trabalho citado.

No quadro abaixo, fazemos o resumo geral dos resultados obtidos, distribuindo os indivíduos examinados, de acôrdo com as respectivas idades. 
RESUMO GERAL DOS RESULTADOS

(Nuc. Col. "B. de Antonina")

\begin{tabular}{|c|c|c|c|}
\hline IDADES & $\begin{array}{l}\text { Positivos para } \\
\text { E. histolytica }\end{array}$ & Negativos & $\begin{array}{c}\text { Total } \\
\text { Examinado }\end{array}$ \\
\hline 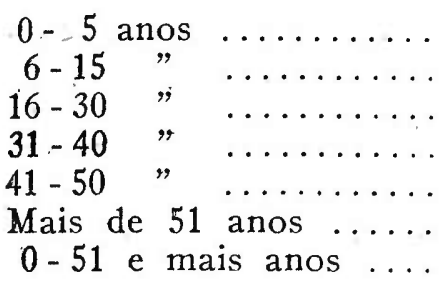 & $\begin{array}{r}5 \\
25 \\
16 \\
9 \\
5 \\
4 \\
64\end{array}$ & $\begin{array}{r}33 \\
117 \\
84 \\
54 \\
18 \\
30 \\
336\end{array}$ & $\begin{array}{r}38 \\
142 \\
100 \\
63 \\
23 \\
34 \\
400\end{array}$ \\
\hline
\end{tabular}

\section{COMENTARIOS}

Não pretendemos, com a apresentação desta pequena nota, tirar qualquer conclusão definitiva a respeito da amebiase em nossos meios rurais. Cremo-nos, porém, autorizado, diante dos resultados a que chegámos nêste nosso inquérito, a dizer que êles são bem um índice de que a Endamoeba histolytica, incidindo em percentagem apreciavel em tais populações, deve contribuir com um coeficiente de grande importância na respectiva nosologia. Com efeito, a incidência de $16 \%$, obtida com um único exame pelo processo de centrifugação e flutuação no sulfato de zinco, faz supor que, fossem os exames repetidos várias vezes entre os negativos, segundo o plano que aconselhamos noutro trabalho (4), tal percentagem ultrapassaria 30. Assim, a amebiase constitue um problema dentre os muitos problemas sanitários que se nos deparam no meio rural.

Digna de menção especial é a incidência de portadores de cistos de $E$. histolytica, com pouca idade, no inquerito a que procedemos. De fato, se considerarmos apenas, no quadro apresentado, os indivíduos em idade escolar (6-15 anos), vemos que, entre 142 examinados nestas condições, 25 revelaram a presença de cistos da endameba patogenica, o que dá o coeficiente de $17,6 \%$.

Aqui fica mais esta nossa modesta contribuição para o conhecimento da incidência da Endamoeba histolytica, entre nós. Necessário se torna à multiplicação de estudos nesse sentido, para termos um đia dados absolutamente seguros que nos permitam avaliar a extensão e conhecer a significação da amebiase, em nosso Estado e em nosso Pais. E isto principalmente porque ha, como vimos, certâs investigações segundo as quais essa protozoose não teria a importância que julgamos ter em nosso meio. A nossa experiência, um tanto longa, na pesquisa da $E$. histolytica, pelas técnicas mais perfeitas e modernas, quer em pequenos inquéritos epidemiológicos, quer na elucidação de diagnósticos, com um acervo de alguns milhares de exames de fézes, já nos autoriza a declarar que, infelizmente, não assiste razão aos que 
querem negar a esse protozoário uma elevada incidência entre 1ós: Continuaremos, porém, as nossas pesquisas, cujos resultados viremos trazendo oportunamente ao conhecimento dos colegas.

\section{SUMÁRIO}

O A. diz, de início, que o problema amebiase, em nosso País, a-pesar-de contribuições valiosas a seu respeito, não está completamente elucidado, pois ainda não dispomos de dados suficientes para aquilatar da importância que essa protozoose deve assumir entre nós. Os estudos até agora levados a cabo em relação ao assunto, ainda que bem conduzidos, são relativamente escassos para a nossa vastidão territorial. Além disso, ha discrepâncias nos resultados a que têm chegado os investigadores do assunto: ao lado de pesquisas, que demonstram ser a amebiase um sério problema para nós, outras existem que, antes, tendem a dar pouca importância à Endamoeba histolytica, em nosso meio. Daí a necessidade de se intensificarem os estudos em relação ao assunto, a exemplo do que se tem feito nos Estados Unidos e na Argentina. A nota que o A. trouxe à Secção se baseia num estudo feito entre os moradores do Núcleo Colonial "Barão de Antonina”, no Município de Itaporanga, Estado de São Paulo. O método de exame de fézes empregado foi o da centrifugação e flutuação no sulfato de zinco, método de Faust et al. O material examinado vinha conservado na solução de formol a $10 \%$. O emprego do formol para a conservação de cistos de protozoários não conta com a simpatia de alguns autores, sob a alegação de que tal conservador não deixaria os cistos em bôas condições para o diagnóstico. O A., porém, do que pôde observar, julgou-se habilitado a concluir que, se, na verdade, o material fresco oferece maiores vantagens para o exame, o conservado em formol, entretanto, dá resultados satisfatórios. No inquérito procedido pelo A. foram examinadas as fézes de 400 indivíduos, entre os quais foram encontrados 64 portadores de $E$. histolytica, o que dá a incidência de $16 \%$. Considerando que, segundo verificação do A., em colaboração com Avila Pires, o método de Faust et al., aplicado uma só vez, num determinado grupo de indivíduos, revela apenas um pouco mais de $50 \%$ dos portadores de cistos de E. histolytica, é de crer que, no grupo em questão, os $16 \%$ se elevariam, no mínimo, a $32 \%$, caso fossem os exames repetidos entre os negativos várias vezes, conforme aconselham o A. e Avila Pires, en trabalho intitulado "Nota sôbre a incidência de portadores de cistos da Endamoeba histolytica (Estudo em 300 presidiários)", trabalho esse lido na reunião de março do corrente ano, na Sociedade de Gastro-Enterologia de São Paulo, e em vias de publicação. Apresenta, em quadro, a incidência da $E$. histolytica, de acôrdo com a idade. 


\section{REFERENCIAS BIBLIOGRÁFICAS}

1 - Young, J. C. - Human intestinal protozoa in Amazonas - Ann. Trop. Med. a. Parasit., 16 (1): 93, 1922.

2 - Faust, E. C., D’Antoni, J. S., Odom, V., Millek, M. J., Peres, C.. Sawitz, W., Thomen, L. F., Tobie, J., e Walker, J. H. - A critical study of laboratory technics for the diagnosis of protozoan cysts and helminth eggs in feces - Am. J. Trop. Med., 18:169-183, 1938.

3 - Faust, E. C., Sawitz, W., Tobie, J., Odom, V., Peres, C., e Lincicome, D. R. - Comparative efficiency of various technics for the diagnosis of protozoa and helminth in feces - Am. J. Parasit., 25:241-262, 1939.

4 - Amaral, A. D. F., e Pires, C. D. A. - Nota sôbre a incidência de portadores de cistos de Endamoeba histolytica (em publicação n'" O Hospital”).

5 - Ficker, M. - Sobre a dysenteria em São Paulo - Ann. paul. med. cir., $5: 335,1915$.

6 - PessôA, S. B. e CORRÊA, C. - Considerações acerca de alguns protozoarios encontrados nas fezes do homem en São Paulo — Folha Médica, Ano VIII (7) : 85-87, abril, 1927.

7 - Vieira, F. B. e Silveira, G. F. - Protozoarios intestinaes no homem da cidade de S. Paulo - São Paulo Médico 2 (1) : 27-35, jan., 1931.

8 - Aragão, R. M. - Febre typhoide, dysenterias e diphteria em João Pessoa - Brasil-Médico, 58:1.058-1.063 e 1.084-1.087, 1938.

9 - Pinro, C. - Sinópse das doenças parasitarias do homem - Tip. Gundlach, Porto Alegre, R. Grande do Sul, 1942.

10 - Pacheco, G. - Sur la fréquence des kystes d'amibes e d'autres protozoaires dans des fèces, apparemment normales, provenant des zones suburbanes et rurales de Rio de Janeiro - Compt. rend. Soc. Biol., 98: 1.5801.561, 1928.

11 - Cunha, A. M. - Protozoarios intestinaes das creanças no Rio de Janeiro - Sciencia Medica, ano 6..$^{\circ}$ março de 1928, pag. 103-109.

12 - Lara, V. e Carvalho, P. E. - Frequencia dos parasitos nas fezes das crianças do Centro de Saúde do Instituto de Higiene - São Paulo Médico, Ano IX, Vol. I, ns. 5 e 6, maio e junho de 1936.

13 - Moraes, R. G. - Portadores de quistos de protozoarios intestinais entre os escolares de Florianopolis - Santa Catarina - Brasil - BrasilCirurgico, 1 (3) : 257-263, nov., 1939.

14 - FAust, E. C.: The prevalence of amebiasis in the Western Hemisphere - The Am. J. of Trop. Med., 22 (1):93, jan. 1942.

15 - Greenway, D. e Castex, M. - Consideraciones parasitologicas y clinicas sobre 2.700 casos de amebiasis intestinal - An. Fac. Cienc. 'Med. de La Plata, vol. 1-2, 1937.

16 - J. A. M. A., 101 (23): 1.806, 1938.

17 - J. A. M. A., 101 (23): 1.819, 1938.

18 - Rosenfeld, G. - Pesquiza de amebas nas fezes. Técnica e conclusões - Rev. clin. de S. Paulo, 2 (2): 47-50, ag., 1937.

19 - D'Antoni, J. S. - Standardization of the iodine stain for wet preparations of intestinal protozoa - Am. J. Trop. Med., 17: 79-84, 1937. 


\section{Preparados Farma ế}

Temos a venda Marcas e Formulas licenciadas e incumbimo-nos de sua compra, venda, ou registro. LICENCIAMOS FORMULAS, PODENDO SER EXAMINADAS POR NOSSO TECNICO FARMACEUTICO OU FORNECER FORMULAS. Legalizamos Laboratorios Farmaceuticos, fazemos quaisquer contratos, de instalação, de exploração, de propaganda, de fabricação.

C ON S ULTEM-N OS SEM COMPROMISSO

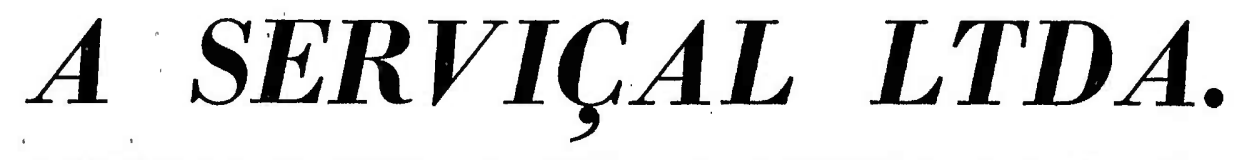

\section{AgENCIAS REUNIDAS RIO DE JANEIRO E S. PAULO}

Diretor Geral: ROMEU RÓDRIGUES

Marcas - Parentes e Licenças de Preparados Farmacêulicos - Comestiveis - Bebidas - Elc.

RIO D E J A N EIRO

Rua São.José n.o 49

$1 .^{\circ}$ Andar

CaIXa POSTAL, 3384

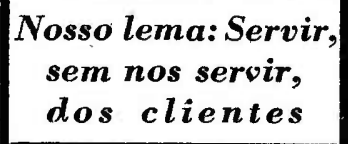

Nosso lema: Servir, dos clientes
$\begin{array}{llllllll}\mathbf{S} & \tilde{\mathbf{A}} & \mathbf{O} & \mathbf{P} & \mathbf{A} & \mathbf{U} & \mathbf{L} & \mathbf{O}\end{array}$

Rua Direita, 64 - 3.० And.

CAIXAS POSTAIS

3631 e 1421

\section{TRATAMENTO DA ASMA}

\section{S A D R E N A L I N H A L A N T E}

Solução concentrada de epinefrina para inhalações.

A inhalação de uma substância capaz de dilatar os brônquios, que ficam contraidos durante o acesso de asma, faz com que desapareçam imediatamente os sintomas.

Este resultado se obtem facilmente com o Isadrenal Inhalante, que pode ser usado em
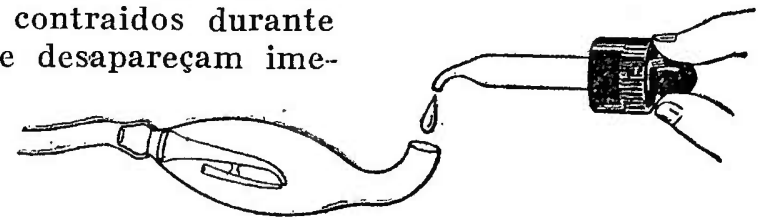
qualquer aparelho para inha-

lação, caso não exista o aparelho Isadrenal Inhalador.

COMO USAR A SOLUGCÃO: - Colocar 20 gotas no aparelho inhalador. Insuflar a pera de borracha, colocando o aparelho verticalmente e dirigido para a boca; ao mesmo tempo inspirar bem fundo, de modo que a vaporização penetre até os pulmões. Poucas insuflações são suficientes para fazer desaparecer o acesso da asma. Terminada a insuflação, o aparelho deve ser fechado com a rolha de borracha e guardado horizontalmente na caixa.

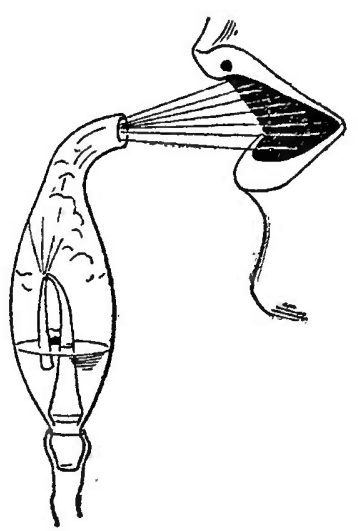

INDÚSTRIA BRASILEIRA DE PRODUTOS QUIMICOS Ltda. MATRIZ: PRAÇA CORNÉlIA, 96 - End. Teleg. "ISA" - Tel.: 5-03.03 - SÃO PAULO FILAL: RUA 13 DE MAIO, 44-A - Sala 1001 - RIO DE JANEIRO 